\title{
A DECOUPLED NUCLEUS IN NGC 1052
}

\section{O.K. SIL'CHENKO}

Sternberg Astronomical Institute

University av. 13, Moscow 119899, Russia.E-mail: olga@sai.msu.su

The central part of NGC 1052 has been observed at the prime focus of the $6 \mathrm{~m}$ telescope with the Multi-Pupil Spectrophotometer equipped with a two-dimensional IPCS. Ninety-nine spectra over the central 14.5 " $x 11$ " are registered in the spectral range of $\lambda \lambda 4700-5400 \mathrm{AA}$ and are used to map ionized-gas velocities (by $[O I I I] \lambda 5007$ ) and to derive radial profiles of absorption-line equivalent width for $M g I \lambda 5175, \mathrm{FeI} \lambda 5270$, and $H_{\beta}$.

The observed position-angle dependence of the central line-of-sight velocity gradient in NGC 1052 is fitted with a cosine law. According to it, the position angle of the gas kinematical major axis is equal to $291^{\circ}$ and perfectly agrees with the photometric major axis $\left(P . A .=113^{\circ}-\right.$ Sparks et al., 1991), and with the stellar kinematical major axis $\left(P . A .=302^{\circ}-\right.$ Davies \& Illingworth, 1986). So our data are fully consistent with an axial symmetry of the very central part of NGC 1052 within a radius of 3 ".

On a radial profile of $M g I \lambda 5175$, a sharp decrease of magnesium-line strength can be seen at the radius of 4 ": 3 points between 0 " and 4 " keep the equivalent width of $5.07 \pm 0.09 \mathrm{~A}$, and the next 4 points up to $10^{\prime \prime}$ stand at the level of $3.52 \pm 0.10 \mathrm{~A}$. The difference is more than $3 \sigma$. By using a model metallicity calibration of $M g b$ by Worthey (1992), a metallicity-break lower limit can be estimated as $0.6 \mathrm{dex}$. A few elliptical galaxies with magnesiumdistinct nuclei are already known (Bender \& Surma, 1992; Sil'chenko et $a l ., 1992)$, but NGC 1052 presents the first case of a RESOLVED distinct nucleus with a definite radius of 4 ".

\section{References}

Bender, R. and Surma, P. (1992) Astron. Astrophys., 258, pp. 250-254

Davies, R.L. and Illingworth, G.D. (1986) Astrophys. J., 302, pp. 234-244

Sil'chenko, O.K., Afanasiev, V.L., Vlasiuk, V.V. (1992) Astron. Zh., 69, pp. 1121-1135

Sparks, W.B., Wall, J.V., Jorden, P.R., Thorne, D.J., and Van Breda, I. (1991) Astrophys. J. Suppl. Ser., 76, pp. 471-524

Worthey, G. (1992) $\mathrm{Ph}$. D. Thesis, California Univ. 